\title{
Impact of chronic respiratory symptoms in a rural area of sub-Saharan Africa: an in-depth qualitative study in the Masindi district of Uganda
}

\section{*Frederik van Gemert', Niels Chavannes ${ }^{2}$, Nahid Nabadda ${ }^{3}$, Simon Luzige ${ }^{4}$, Bruce Kirenga ${ }^{4}$, Celeste Eggermont ${ }^{5}$, Corina de Jong', Thys van der Molen'}

\author{
1 Department of General Practice, University of Groningen, University Medical Centre Groningen, Groningen, The Netherlands \\ 2 Department of Public Health and Primary Care, Leiden University Medical Centre, Leiden, The Netherlands \\ ${ }^{3}$ Multi Consults Research Ltd, Kampala, Uganda \\ ${ }^{4}$ Department of Medicine, Mulago Hospital, Kampala, Uganda \\ ${ }^{5}$ Faculty of Medicine, University Medical Centre Utrecht, Utrecht, The Netherlands
}

Original received 19th December 2012; resubmitted 3rd March 2013; revised 11th April 2013; accepted 22nd April 2013; online 2nd July 2013

\begin{abstract}
Background: Chronic obstructive pulmonary disease (COPD), once regarded as a disease of developed countries, is now recognised as a common disease in low- and middle-income countries. No studies have been performed to examine how the community in resourcepoor settings of a rural area in sub-Saharan Africa lives with chronic respiratory symptoms.

Aims: To explore beliefs and attitudes concerning health (particularly respiratory illnesses), use of biomass fuels, tobacco smoking, and the use of health services.

Methods: A qualitative study was undertaken in a rural area of Masindi district in Uganda, using focus group discussions with 10-15 members of the community in 10 randomly selected villages.

Results: Respiratory symptoms were common among men, women, and children. In several communities respiratory symptoms were stigmatised and often associated with tuberculosis. Almost all the households used firewood for cooking and the majority cooked indoors without any ventilation. The extent of exposure to tobacco and biomass fuel smoke was largely determined by their cultural tradition and gender, tribal origin and socioeconomic factors. Many people were unaware of the damage to respiratory health caused by these risk factors, notably the disproportionate effect of biomass smoke in women and children.

Conclusions: The knowledge of chronic respiratory diseases, particularly COPD, is poor in the rural community in sub-Saharan Africa. The lack of knowledge has created different beliefs and attitudes concerning respiratory symptoms. Few people are aware of the relation between smoke and respiratory health, leading to extensive exposure to mostly biomass-related smoke.

(C) 2013 Primary Care Respiratory Society UK. All rights reserved.

F van Gemert et al. Prim Care Respir J 2013; 22(3): 300-305

http://dx.doi.org/10.4104/pcrj.2013.00064
\end{abstract}

Keywords COPD, biomass fuel, sub-Saharan Africa, qualitative study, respiratory symptoms

\section{See linked editorial by Namukwaya et al. on pg 265}

\section{Introduction}

In developing countries chronic obstructive pulmonary disease (COPD) is an increasing health problem, a fact recognised only a decade ago by international health authorities. ${ }^{1.3}$ At present COPD is the fourth leading cause of death, and by 2025 it is predicted to become the third, by which time it will surpass HIVIAIDS in Africa. ${ }^{4}$
Tobacco smoking has traditionally been considered the main risk factor responsible for the development of COPD. However, in the last decade a growing number of studies have suggested that other risk factors also have an important role in the development of COPD, such as asthma, air pollution, occupational exposures, socioeconomic status, (treated) tuberculosis, and other populationattributable risk factors. ${ }^{5.8}$ Indoor air pollution caused by biomass fuel use (wood, crop residues, twigs, dried dung and charcoal) has

\footnotetext{
* Corresponding author: Mr Frederik van Gemert, University Medical Centre Groningen, Department of General Practice, A Deusinglaan 1, P O Box 196, 9700 AD Groningen, The Netherlands. Tel: +31 503637584 Fax: +31 503632964 E-mail: frgemert@xs4all.nl
} 
been shown to be a strong and independent risk factor for COPD. ${ }^{5,79,10}$ Over three billion people - and $90 \%$ of rural households in developing countries - rely on biomass fuel as their main source of domestic energy." The majority of these solid fuels are burnt in poorly functioning cooking stoves or open fires with inefficient combustion and in the presence of poor ventilation, leading to extremely high levels of indoor pollution. ${ }^{9-12}$

In sub-Saharan Africa the knowledge of COPD is very poor. ${ }^{13}$ The treatment for chronic respiratory diseases (CRDs) is often focused only on ad hoc treatment of acute exacerbations and tuberculosis (TB). Diagnostic tests such as spirometry or even peak flow measurements are only rarely available. Inhaled medication is often limited and/or not affordable. ${ }^{14,15}$ Nevertheless, respiratory symptoms are among the major causes of consultation at healthcare centres. ${ }^{1,16,17}$

To our knowledge, no studies have been carried out to examine how the community in resource-poor settings of a rural area in subSaharan Africa lives with COPD. One of the low-income countries in sub-Saharan Africa is Uganda, which has a population of about 33 million people. ${ }^{18}$ The recently published 'Health Sector Strategic Plan III 2010-2015' from the Ministry of Health in Uganda does not mention COPD at all. ${ }^{19}$ Around $78 \%$ of the population live in rural areas; $86 \%$ of this rural population use wood for cooking and $10 \%$ use charcoal. ${ }^{18}$

The aim of this qualitative study was to explore the beliefs and attitudes in the rural community concerning health (particularly chronic respiratory illnesses), use of biomass fuels, tobacco smoking, and the use of health services. Focus group discussions (FGDs) were used to find answers to the following questions:

- How does the community cope with respiratory symptoms?

- Does gender influence their behaviour?

- What are the cooking habits and the traditions of the community?

- Are the cultural taboos and cultural practices affected by their tribal origin?

- What is the knowledge of the community concerning the risk factors of CRDs?

\section{Methods}

\section{Setting}

The FGDs, undertaken in March 2011, targeted Masindi district, located in the mid-western part of Uganda. The population was predominantly rural with only $5.4 \%$ of the residents living in urban areas. The district population in 2010 was estimated at approximately 370,000. Masindi district has a diverse ethnic composition of 55 tribes, with the dominant tribes - the Banyoro and the Lugbara - forming about $60 \%$ of the population. ${ }^{18}$ Ten villages within the three counties were randomly selected, three each in the smaller counties and four in the largest. Half of the villages were in tobacco growing areas.

\section{Data collection}

Prior to the FGDs, all the villages were visited and the village leaders were consulted to obtain community consent after giving the relevant information about the purpose of the study. For the FGDs,

\section{Box 1. Focus group interview questions}

A. Knowledge and attitude

1. How is the knowledge, attitude and practice when it comes to respiratory symptoms?

2. How is the knowledge and attitude of chronic respiratory diseases?

3. How is the knowledge of risk factors such as biomass fuel use and tobacco smoke?

4. How many households have adopted intervention programs?

5. How is the health-seeking behaviour for respiratory symptoms?

B. Biomass fuel use

1. What type of fuel is mainly used for cooking and heating?

2. Where is the cooking usually done (indoor or outdoor, rooms used for living/sleeping)?

3. What type of ventilation is used to remove smoke from their homes?

5. Who are most exposed to biomass smoke?

6. What is the duration of exposure to biomass smoke?

7. How does culture and tradition affect the exposure to biomass smoke?

\section{Tobacco use}

1. What are the most common tobacco products?

2. What is the average number of smokers in the community?

3. How does culture and tradition affect their smoking behaviour?

we wanted to have four groups of women aged $>30$ years, three groups of men aged $>30$ years, and three mixed groups of men and women aged 15-25 years. The separation was intended to capture specific information, beliefs, and attitudes of varying community clusters independently. In each village the village leader was asked to select randomly 10 members of the community for a specific group. All the FGDs were led by the same sociologist from Uganda. There were many different languages spoken by the tribes, of which Lunyoro and Swahili were most dominant. The sociologist spoke Lunyoro and received help from the village leader as interpreter whenever other languages were spoken. Each group had 10-15 participants. In some villages the village leader could not exclude several participants for social reasons. The FGDs lasted approximately one hour and all the discussions were audiotaped. The interview questions had three main themes: knowledge and attitude; biomass fuel use; and tobacco use (Box 1).

\section{Data analysis}

The interviews were translated into English and transcribed verbatim. The transcriptions were read several times independently by FvG, CdJ, and TvdM to get an overall picture, and then entered into NVivo 9, a qualitative data management program. With full agreement, a thematic framework was generated for the key themes and sub-themes. This was used to code all transcripts and to explore and compare themes between interviews. This process was undertaken by FvG and was reviewed frequently with $\mathrm{CdJ}$ and TvdM to ensure trustworthiness and validity of data analysis.

\section{Ethical considerations}

This qualitative study was approved by the local medical ethics 
committee, the Uganda National Council for Science and Technology.

\section{Results}

\section{Chronic respiratory symptoms}

In most of the FGDs the word 'asthma' was known, although they did not understand the implication of this disease. The word 'COPD', however, was totally unknown. Most participants of the FGDs knew TB as a serious disease.

Chronic cough seemed to be common in these rural areas, not only among smoking men but also among non-smoking women and children. It was often associated with TB and viewed as contagious.

"There is a fear of TB: as long as somebody does not know the cause of his or her coughing, we will be cautious relating with them because it might be TB". (FGD, women)

In many areas chronic cough was stigmatised: in their homes the utensils were kept aside, at times even when their diagnosis for TB was negative. In certain communities a person with chronic cough could even become an outcast. During the village meetings they were kept at a distance, in isolation from the rest of the village members.

"It is the fear of stigma that makes them keep their illnesses to themselves. When people become aware you have any of these diseases, you cannot sit close to people like here". (FGD, youth)

The situation was different in tobacco-growing areas where the communities were so accustomed to living with chronic cough that they did not consider it a health problem. In these areas the stigma was limited.

"We don't mind smoker's coughing; we know it is a result of their smoking and not contagious". (FGD, men)

"Women with chronic cough are not as accepted as men. As a man I don't want my wife to have a chronic cough; I give

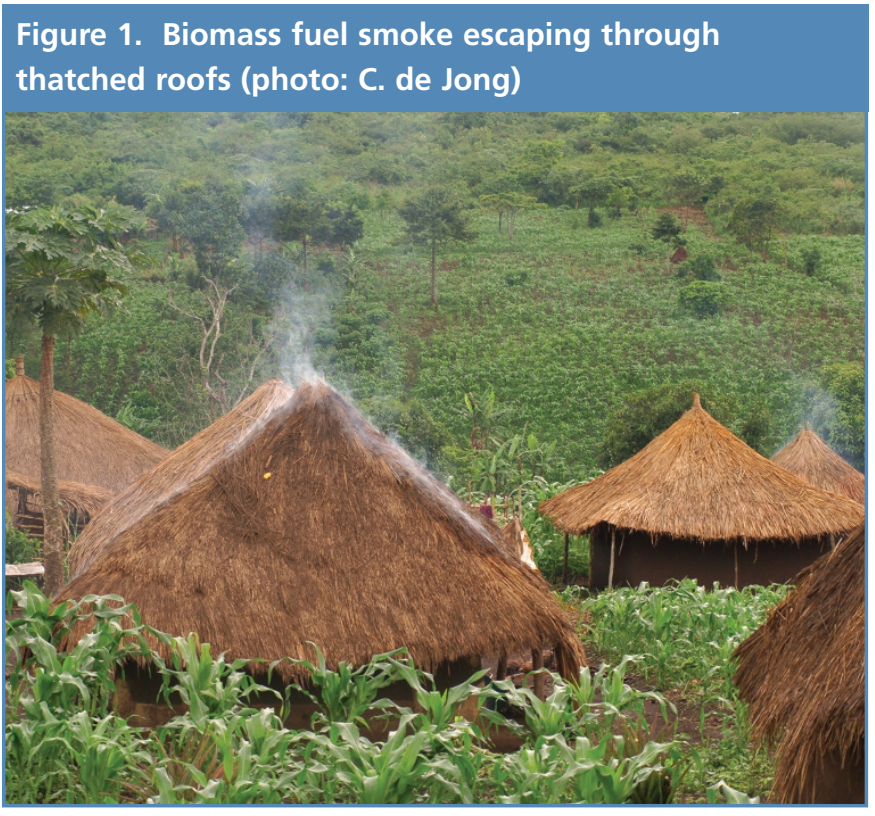

her herbs or get her medical treatment because it becomes irritating". (FGD, men)

In most of the focus groups wheezing was considered a serious condition worth seeking medical care. Wheezing was often associated with asthma. In several villages asthma was thought to be contagious. In one village the adults had tried to keep their wheezing child a secret, except for direct family members.

Shortness of breath was seen among adults aged $>40$ years, particularly in areas where the villagers grew tobacco.

"There is a lady with shortness of breath. She has been treated at the health centre but she hasn't improved. She

has given up hope of getting better". (FGD, youth)

Occasionally the community considered shortness of breath as being contagious too. Most of the time patients refrained from seeking medical care unless their symptoms deteriorated in a very short time.

\section{Biomass fuel exposure}

Almost all the households in the rural areas used firewood for cooking; a small percentage used charcoal, particularly in urban areas. Charcoal was too expensive for most communities. In addition, firewood gave the food a more favourable taste. Crop residues and dung were barely used. The majority cooked indoors in the kitchen, using an open fire with three rocks as support for the pot or griddle. There were no chimneys and there was hardly any ventilation. Some people used their sleeping space for cooking, exposing the whole family to biomass smoke. Others, who could afford more, had a separate kitchen in their homes. Occasionally the fire was used for warmth

"Children love sitting in the kitchen for warmth. As soon it gets dark, they run to their mother's kitchen". (FDG, men) A few people had well aerated kitchens, but these were considered incomplete and undesirable by a large part of the community because of their inability to be used for storage. Rain (particularly during the wet seasons) and wind made cooking outside problematic.

Figure 2. Traditional open fire used for cooking, leading to high levels of indoor pollution (photo: F.A. van Gemert)

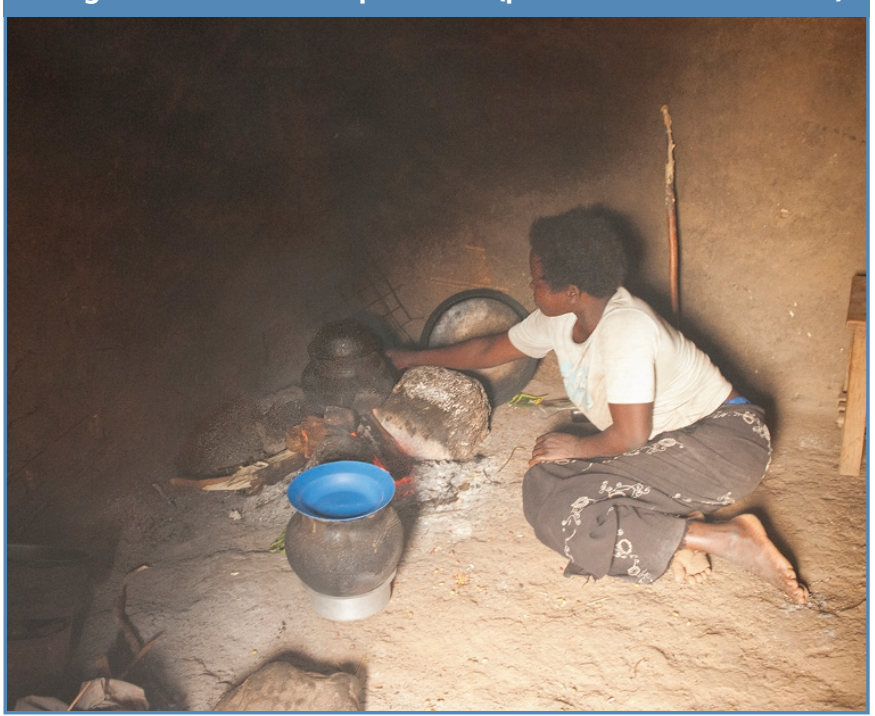


"When you cook outside, the wind blows the fire around; the wood gets burnt out when the food is not ready". (FGD, women)

"You also use a lot of firewood because of the wind, yet it is scarce". (FGD, women)

Particularly during the harvest and wet seasons, the different tribal groups ate sweet potatoes, matooke (steamed green bananas) and dried beans, all taking about three hours to cook. All the household chores were the responsibility of the women, whether they were ill or not. The tradition of initiating young girls aged 6-12 years into womanhood exposed the children to biomass smoke at a young age.

"That is how we learnt how to cook ourselves. When the children grow older, it becomes harder to teach them because they have to go to school". (FGD, youth)

In total, women were exposed to biomass smoke for about six hours per day, most of the time in the afternoon and evening after working in the fields in the morning. A few women in the communities did realise firewood smoke affected their health.

"I am afraid I might get health problems as a result of

firewood smoke. That smoke might be the reason why some people have breathing problems and chronic cough". (FGD, women)

It was almost impossible for women to change the age-old cooking tradition as they were lowest in societal position. Many women still cooked inside with their babies in the kitchen, particularly when they were breast feeding. Children under the age of 5 were often close by their mother when she was cooking. During one of the male FGDs they were talking about a woman with a chronic cough and the impact of using firewood for cooking. One man's concern was:

"Who will cook for this woman's husband if she has to limit

her exposure to smoke? How will he survive?" (FGD, men)

Men rarely did household chores as it was considered a sign of weakness in rural communities. The men therefore reacted differently:

"We can tell you about tobacco smoking, but we know nothing about kitchen smoke: that is women's work." (FGD, men)

"We do not cook and we have no idea that firewood smoke affects those who cook." (FGD, men)

\section{Tobacco}

In tobacco-growing areas most men and many of the elderly women smoked tobacco. It was their culture and they had easy access to tobacco products. Cannabis was smoked predominantly by young men. Young children smoked too, picking up the pieces thrown away by their fathers. Most of the adults smoked tobacco leaves wrapped in paper (simonko), some mixed tobacco with ashes and placed it under their tongues, and others - especially men who could afford it - smoked cigarettes. Many men believed that cigarettes were safe; women often did not agree.

"It is tobacco leaves that are not good; cigarettes are not as hazardous". (FGD, men)

"Many people who smoke simonko and cigarettes have chronic cough". (FGD, women)
Men smoked anywhere they wanted and smoked indoors in the presence of children, exposing their families to tobacco smoke every night. They could not be challenged as they were the head of the family and could do whatever they wanted.

"My husband smokes in bed in the middle of the night claiming he owns the house and can do whatever he wants, even smoking under the mosquito net". (FGD, women)

"My husband told me if I am bothered by his smoking, I am

free to leave so that he can bring his other wife". (FGD, women)

"I smoke with my mouth, not yours ..." (FGD, men)

Tobacco leaves were dried in sealed houses using an open fire. Both adults and children kept watch inside to ensure the leaves did not catch fire. Some adults had spent more than 30 years being exposed to that smoke.

\section{Medical care}

The communities often used local herbs as treatment (boiled mango leaves), although the use of traditional healers was denied in all the FGDs. Sometimes they bought syrups at the dispensaries, but these were rather expensive.

"Sometimes you do not have the money to buy drugs for the prescription; that is why we keep quiet with our illness and do not seek medical treatment". (FGD, women)

The communities in the urban areas often felt that the medical professionals favoured town people over people from the villages. Their trust in health centres was low, particularly when it concerned chronic respiratory symptoms. An occasional 'TB-negative' diagnosis was frustrating for the community because this meant the healthcare centres had no possibility to treat them.

"My wife coughs a lot. She was tested for TB and the results were negative. She has been treated without improvement". (FGD, men)

"My husband, a former addict to tobacco, has a chronic cough. He has shortness of breath and sometimes almost suffocates. He had treatment from the health centre but it has not been effective". (FGD, women)

The health centres sometimes only had a few essential drugs in stock such as antibiotics, paracetamol, and antimalaria drugs. In Masindi district, salbutamol tablets were only available at the hospital. Inhaled medication was not available at all.

\section{Discussion}

\section{Main findings}

The results of the focus group discussions (FGDs) reveal that many people of all ages have respiratory symptoms. The general lack of knowledge on CRDs, particularly COPD, has created different beliefs and attitudes towards respiratory symptoms in the different communities. This varies from acceptance in communities with a high incidence and stigmatisation in low incidence communities. The connection between TB and chronic respiratory symptoms is often made.

Each tribal group has distinctive practices when it comes to staple food, cooking tradition, sleeping areas, and smoking habits, all of which largely affect levels of exposure to biomass fuel smoke and 
tobacco smoke. Generally speaking, wood is the major source of biomass fuel for cooking and heating. The poorest people in the rural areas are most exposed to biomass fuel smoke as they cannot afford a separate cooking place. Societal roles are largely determined by gender, with the result that women are much more exposed to biomass smoke than men, starting at a young age. Growing tobacco is for several tribes the main source of income and hence is easily accessible, resulting in a high smoking prevalence.

\section{Strengths and limitations of this study}

This is the first qualitative study to explore attitudes and beliefs on respiratory symptoms, tobacco smoking, biomass fuel use, and use of health services in a rural area of a sub-Saharan country.

At first the FGDs were undertaken very carefully as a lack of clarity existed regarding cultural sensitivities in relation to specific questions. The initial discussions were therefore open-structured. In the course of the FGDs we had the opportunity to develop a semistructured protocol for the discussions by analysing the field notes made by the sociologist manually during the fieldwork. This allowed us to focus on specific issues raised by the participants in the next FGDs.

As a translator and interviewer, the sociologist was offered significant opportunities for close attention to address cultural meanings during the FGDs. The field notes and the translations hereafter were regarded as a check to the validity of interpretations.

A drawback was the use of an interpreter in several of the villages visited. Even though all the village leaders spoke English and the primary language of the village, they were not always familiar with medical terminology. We therefore endeavoured to formulate short and simple questions. On several occasions words and concepts were demonstrated to make sure people understood.

\section{Interpretation of findings in relation to previously} published work

Chronic respiratory symptoms such as chronic cough, sputum, shortness of breath, wheeze, and even serious respiratory infections seem to be very common in the rural district of Masindi. Tobacco smoking and indoor biomass fuel use could be major contributing factors to these symptoms in men, women, and children.1,10,20-22 Many people, however, are still unaware of the damage to respiratory health caused by smoking tobacco and biomass fuel use. ${ }^{1.8}$ The use of biomass fuel is not only determined by their cultural tradition but also by socioeconomic factors such as poverty and living conditions..$^{10}$ The nature of the communities also determines healthcare-seeking behaviour - both traditional (local herbs) and western (dispensaries and health centres) - most of the time with a lack of successful results. Implications for future research, policy and practice Exposure to tobacco smoke as well as biomass fuel smoke may cause a high incidence of COPD in these communities. Wood smoke exposure has been shown to be a major contributing factor to the development of COPD, causing a preventable burden in sub-Saharan Africa. ${ }^{79,10,12}$ Unfortunately, COPD and its main risk factors receive insufficient attention from the healthcare community, government officials, patients, and their families. A major priority should be increasing the knowledge of COPD in the various communities, and promoting awareness among healthcare workers about the detrimental health effects of these risk factors.

More research is needed in sub-Saharan Africa on the prevalence of COPD and its risk factors. It is critically important that the social, cultural, and economic context is taken into account when a study is designed, and that it should contribute directly to the strengthening of local knowledge of COPD.

\section{Conclusions}

The knowledge of CRDs, particularly COPD, in this area of Uganda and probably many other rural areas in sub-Saharan Africa - is poor. The lack of knowledge has created different beliefs and attitudes. The majority of the community uses biomass fuels to cook and is unaware of the damage caused by tobacco and biomass smoke. The extent of exposure to these risk factors is largely determined by gender, cultural tradition, tribal origin, and socioeconomic status. Further research on the prevalence of COPD and its risk factors might help to avoid the high prevalence of COPD in sub-Saharan Africa in the future.

\section{Handling editor Maureen George}

Acknowledgements We would like to thank all the participants in the villages who took part in the focus group discussions.

Conflicts of interest The authors declare that they have no conflicts of interest in relation to the article. NC is an Associate editor of the PCRJ, but was not involved in the editorial review of, nor the decision to publish, this article.

Contributorship FvG, as main researcher, designed and organised the qualitative study, worked on the thematic analysis of the qualitative data and wrote the manuscript. NC participated in the design and organisation of the study, provided feedback and was involved in generation of the manuscript. NN led the focus group discussions as she spoke their language and wrote the transcripts. SL and BK contributed to the sampling and methodology and were involved in the generation of the manuscript. $\mathrm{CdJ}$ contributed particularly to the qualitative analysis and was involved in the generation of the manuscript. CE made a contribution to acquisition of the data, was involved in analysing the field notes during the fieldwork and in generating the first draft of the manuscript. TvdM participated in the design of the study, contributed to the qualitative analysis of the data, provided feedback and was involved in generating the manuscript. All authors read and approved the final manuscript. FvG, NN, and CE were active in Uganda during the study. FvG is the guarantor.

Funding This qualitative study was funded by the International Primary Care Respiratory Group, who had no role in the design, conduct, analysis, or reporting of the study or in the decision to submit the manuscript for publication.

\section{References}

1. Aït-Khaled N, Enarson DA, Chiang C. COPD management. Part II. Relevance for resource-poor settings. Int J Tuberc Lung Dis 2008;12(6):595-600.

2. Chan-Yeung M, Ait-Khaled N, White N, Ip MS, Tan WC. The burden and impact of COPD in Asia and Africa. Int J Tuberc Lung Dis 2004;8(1):2-14

3. World Health Organization. Global Alliance against Respiratory Diseases: Action Plan 2008-2013. Geneva: World Health Organization, 2008.

4. World Health Organization. World Health Statistics. Geneva: World Health Organization, 2008

5. Bousquet J, Dahl R, Khaltaev N. Global alliance against chronic respiratory diseases Allergy 2007;62(3):216-23. http://dx.doi.org/10.1111/j.1398-9995.2007.01307.x

6. Mannino DM, Buist AS. Global burden of COPD: risk factors, prevalence, and future trends. Lancet 2007;370(9589):765-73. http://dx.doi.org/10.1016/S0140-6736(07)61380-4

7. Salvi S, Barnes PJ. Is exposure to biomass smoke the biggest risk factor for COPD globally? Chest 2010;138(1):3-6. http://dx.doi.org/10.1378/chest.10-0645

8. Buist AS, Vollmer WM, McBurnie MA. Worldwide burden of COPD in high- and lowincome countries. Part I. The Burden of Obstructive Lung Disease (BOLD) initiative. Int J Tuberc Lung Dis 2008;12(7):703-08.

9. Kurmi OP, Lam KB, Ayres JG. Indoor air pollution and the lung in low- and mediumincome countries. Eur Respir J 2012;40(1):239-54

http://dx.doi.org/10.1183/09031936.00190211 
10. Perez-Padilla R, Schilmann A, Riojas-Rodriguez H. Respiratory health effects of indoor air pollution. Int J Tuberc Lung Dis 2010;14(9):1079-86

11. Kurmi OP, Semple S, Simkhada P, Smith WC, Ayres JG. COPD and chronic bronchitis risk of indoor air pollution from solid fuel: a systematic review and meta-analysis Thorax 2010;65(3):221-8. http://dx.doi.org/10.1136/thx.2009.124644

12. Viegi G, Maio S, Pistelli F, Baldacci S, Carrozzi L. Epidemiology of chronic obstructive pulmonary disease: health effects of air pollution. Respirology 2006;11(5):523-32. http://dx.doi.org/10.1111/j.1440-1843.2006.00886.x

13. van Gemert F, van der Molen T, Jones R, Chavannes N. The impact of asthma and COPD in sub-Saharan Africa. Prim Care Respir J 2011;20(3):240-8. http://dx.doi.org/10.4104/pcri.2011.00027

14. Martins P, Rosado-Pinto J, do Ceu Teixeira M, et al. Under-report and underdiagnosis of chronic respiratory diseases in an African country. Allergy 2009;64(7):1061-7. http://dx.doi.org/10.1111/j.1398-9995.2009.01956.x

15. Mehrotra A, Oluwole AM, Gordon SB. The burden of COPD in Africa: a literature review and prospective survey of the availability of spirometry for COPD diagnosis in Africa. Trop Med Int Health 2009;14(8):840-8. http://dx.doi.org/10.1111//.1365-3156.2009.02308.x

16. Aitt-Khaled N, Enarson DA, Ottmani S, El Sony A, Eltigani M, Sepulveda R. Chronic airflow limitation in developing countries: burden and priorities. Int I Chron Obstruct Pulmon Dis 2007;2(2):141-50.

17. World Health Organization. Global Surveillance, Prevention and Control of Chronic Respiratory Diseases: A Comprehensive Approach. Geneva: World Health Organization, 2007

18. Male-Mukasa JB. Uganda National Household Survey, Uganda Bureau of Statistics, 2010.

19. Orichi Orach S. Health Sector Strategic Plan III, 2010/11-2014-15. Government of Uganda, Ministry of Health.

20. Dherani M, Pope D, Mascarenhas M, Smith KR, Weber M, Bruce N. Indoor air pollution from unprocessed solid fuel use and pneumonia risk in children aged under five years: a systematic review and meta-analysis. Bull World Health Organ 2008;86(5):390-8C. http://dx.doi.org/10.2471/BLT.07.044529

21. Naeher $L P$, Brauer $M$, Lipsett $M$, et al. Woodsmoke health effects: a review. Inhal Toxicol 2007;19(1):67-106. http://dx.doi.org/10.1080/08958370600985875

22. Rumchev K, Spickett JT, Brown HL, Mkhweli B. Indoor air pollution from biomass combustion and respiratory symptoms of women and children in a Zimbabwean village. Indoor Air 2007;17(6):468-74.

\section{Available online at http://www.thepcrj.org}

